\title{
Prevention and treatment strategies for type 2 diabetes based on regulating intestinal flora
}

\author{
Dandan Xie ${ }^{\S}$, Xiaotong Zhao ${ }^{\S}$, Mingwei Chen* \\ Department of Endocrinology, the First Affiliated Hospital of Anhui Medical University, Hefei, Anhui, China.
}

\begin{abstract}
SUMMARY Diabetes along with related comorbidities associated with high disability rates severely threatens human health. The etiology of diabetes is complex. Genetics, environmental factors, eating habits, drug usage, aging, and lack of movement play important roles in the development of diabetes. Intestinal flora is reportedly closely related to the occurrence and development of type 2 diabetes. Herein, we review changes in abundance and proportion of intestinal flora in patients with type 2 diabetes and regulation of intestinal flora through diet, drugs, and surgery to prevent and treat type 2 diabetes. A more appropriate clinical diagnosis and treatment plan could be made considering changes in intestinal flora in the future.
\end{abstract}

Keywords type 2 diabetes mellitus, intestinal microbiota, insulin resistance, research progress

\section{Introduction}

With the development of the economy and society, the global urbanization process has accelerated, and people's lifestyle and dietary habits have changed dramatically. Excessive salt, sugar, and fat in the diet increase the incidence of chronic metabolic diseases, such as obesity and diabetes. According to the latest data released by the International Diabetes Federation, there were approximately 463 million people aged 2079 years with diabetes worldwide in 2019. China has the highest number of adults with diabetes (116.4 million), accounting for a quarter of the world's diabetes-affected population. According to forecasting models, diabetes will affect 700.2 million people by 2045 (1). Type 2 diabetes mellitus (T2DM) accounts for more than $90 \%$ of the total number of diabetic patients. Hence, it is of great significance to study the pathophysiological mechanisms and effective prevention and treatment of T2DM.

T2DM is a metabolic syndrome caused by the combined effects of genetic and environmental factors, and is characterized by an absolute or relative deficiency of insulin secretion and a decrease in insulin sensitivity in target organs. Glucose metabolism disorder is the primary manifestation of T2DM, followed by metabolic disorders of fat, protein, water, and electrolytes. Insulin resistance and dysfunction of islet $\beta$ cells are considered to be the leading causes of the occurrence and development of T2DM (2). The intestinal flora is known as "the second human genome". As an internal environmental factor of the body, it plays a vital role in regulating metabolism, immunity, inflammation, and other physiological and pathological processes. There is increasing evidence that abnormal intestinal flora is closely associated with the occurrence and development of T2DM (3).

More than 1,000 species of bacteria inhabit the human gut, and the total number is approximately $10^{14}$, which is 10 -fold more than the number of human cells. These microbes weigh up to $1.2 \mathrm{~kg}$ in total and account for about $80 \%$ of microbes in the human body. Bacteroidetes and Firmicutes are the two main phyla, followed by Actinomycetes, Proteobacteria, and Verrucomicrobia (4). Depending on their relationship with the host, intestinal flora can be divided into commensal bacteria, opportunistic pathogens, and harmful bacteria. In the physiological state, these organisms are mutually dependent and restricted. They have a symbiotic relationship with the human body and maintain a dynamic balance. Furthermore, commensals are a component of the natural defense line to maintain human health. When pathological factors break this balance, many diseases occur. Herein, we review the characteristics of intestinal flora in patients with T2DM and strategies for preventing and treating T2DM by regulating intestinal flora to provide a relevant reference for clinical diagnosis and treatment of diabetes.

\section{Differences in intestinal flora between T2DM and non-diabetic populations}

Although it is still uncertain whether there is a causal 
Table 1. Differences in the intestinal flora among patients with T2DM, prediabetic population, and normal population in some studies

\begin{tabular}{|c|c|c|c|}
\hline Study & Intestinal flora & Patients with T2DM & Prediabetic population \\
\hline \multirow[t]{3}{*}{ Zhang et al. (5) } & Akkermansia muciniphila and Faecalibacterium prausnitzii & - & $\downarrow$ \\
\hline & Bacteroides & $\downarrow$ & - \\
\hline & Verrucomicrobia & $\downarrow$ & $\downarrow$ \\
\hline Egshatyan et al. (6) & Blautia and Serratia & $\uparrow \uparrow$ & $\uparrow$ \\
\hline \multirow[t]{2}{*}{ Larsen et al. (7) } & Firmicutes & $\downarrow$ & - \\
\hline & Proteobacteria and Bacteroidetes & $\uparrow$ & - \\
\hline Wu et al. (8) & Bifidobacterium and Bacteroides vulgatus & $\downarrow$ & - \\
\hline \multirow[t]{2}{*}{ Sedighi et al. (10) } & Lactobacillus & $\uparrow$ & - \\
\hline & Bifidobacterium & $\downarrow$ & - \\
\hline \multirow[t]{2}{*}{ Hartstra et al. $(13,14)$} & Roseburia, Eubacterium hallii, and Faecalibacterium prausnitzii & $\downarrow$ & - \\
\hline & Lactobacillus gasseri, Streptococcus mutans, and Escherichia coli & $\uparrow$ & - \\
\hline
\end{tabular}

$\uparrow:$ The abundance of the intestinal flora increased in patients with T2DM/prediabetic population. $\downarrow$ :The abundance of the intestinal flora decreased in patients with T2DM/prediabetic population. - :Not mentioned.

relationship between intestinal flora alteration and T2DM, the changes in the intestinal flora in patients with T2DM have been confirmed (Table 1). Zhang et al. found that the abundance of butyric acidproducing bacteria, such as Akkermansia muciniphila and Faecalibacterium prausnitzii in the normal population was higher than that in the prediabetic population, and the abundance of Bacteroides in patients with T2DM was only half of that in the normal population and prediabetic population. The abundance of Verrucomicrobia in the prediabetic population and patients with T2DM was significantly lower than that in the normal population, and it may be a potential marker of T2DM (5). Egshatyan et al. reported that the abundance of Blautia and Serratia in the gut of the prediabetic population was lower than that in patients with T2DM, and people with normal glucose tolerance have the lowest abundance (6). Larsen et al. used realtime quantitative PCR to analyze the fecal flora in 18 patients with T2DM and 18 non-diabetic individuals and found that the abundance of Firmicutes decreased in patients with T2DM, whereas the abundance of Proteobacteria and Bacteroidetes increased. Besides, the ratio of Bacteroidetes to Firmicutes significantly positively correlated with blood glucose concentration (7). Wu et al. found that the abundance of Bifidobacterium and Bacteroides vulgatus in patients with T2DM was significantly lower than that in nondiabetic individuals (8). Karlsson et al. isolated the fecal microbiota from 53 patients with T2DM, 49 people with impaired glucose tolerance, and 43 healthy European women for metagenomic sequencing. They found that compared with the intestinal flora in non-diabetic individuals, 4 Lactobacillus species and 5 Clostridium species were increased and decreased, respectively, in diabetic patients. Lactobacillus positively correlated with blood glucose and glycosylated hemoglobin
(HbA1c), whereas Clostridium negatively correlated with blood glucose, HbAlc, insulin, C-peptide, and triacylglycerol, and positively correlated with adiponectin and high-density lipoprotein (HDL) (9). Sedighi et al. analyzed the microbiome in fecal samples of patients with T2DM and normal populations. They confirmed that intestinal flora of patients with T2DM had a high abundance of Lactobacillus, whereas abundance of Bifidobacterium in healthy individual's intestinal flora was relatively high (10). Pedersen et $a l$. analyzed the difference in serum metabiome and metagenome between 75 patients with T2DM and 291 healthy individuals. They found that when the proportion of Prevotella copri and Bacteroides vulgatus increased, the content of branched-chain amino acids (BCAAs) in serum increased, which induced insulin resistance and aggravated impaired glucose tolerance (11). Lambeth et al. studied the intestinal microbiota characteristics in patients with prediabetes or T2DM and healthy individuals. Compared to patients with T2DM, the abundance of Chloracido members in the prediabetic group was high, and an unknown genus of Pseudonocardiaceae was identified in the prediabetic group. The abundance of Collinsella and an unknown genera of family Enterobacteriaceae in patients with T2DM significantly increased compared with that of the other groups (12). Hartstra et al. reported that the abundance of Roseburia, Eubacterium hallii, and Faecalibacterium prausnitzii decreased in patients with T2DM, whereas that of Lactobacillus gasseri, Streptococcus mutans, and Escherichia coli increased $(13,14)$. Reitmeier et al. analyzed the correlation between rhythmic changes in intestinal microbes and incidence of T2DM. The study involved fecal flora data from more than 4000 people in three German cohorts. It was found that both diversity of intestinal flora and relative abundance of specific flora fluctuated 
at a periodicity of $24 \mathrm{~h}$, and 13 operational taxonomic units (OTUs) that affect microbial rhythm disorders in T2DM were identified, which can accurately identify and predict T2DM (15). Sroka-Oleksiak et al. analyzed the duodenal flora and multiple clinical indicators of 17 obese individuals, 22 obese patients with T2DM, and 27 healthy individuals, and the results suggested that Bifidobacterium may be a biomarker for the occurrence and development of T2DM and obesity in the future (16). Gurung et al. summarized 42 studies on the relationship between intestinal flora and T2DM and concluded that Bacteroides, Faecalibacterium, Akkermansia, and Roseburia negatively correlated with T2DM, whereas Ruminococcus, Fusobacterium, and Blautia positively correlated with T2DM (3). Thus, the number and diversity of intestinal flora in patients with T2DM undergo different degrees of changes, and a regular analysis of the changes in intestinal flora has realistic directive significance.

\section{Prevention and treatment of T2DM based on regulating intestinal flora (Figure 1)}

\subsection{Probiotics and prebiotics}

Probiotics are a class of active microorganisms that have beneficial effects on host health. They affect host energy and substance metabolism by improving the host microbiota (17). At present, there are three main types of probiotics: strictly anaerobic Bifidobacterium, aerotolerant Lactobacillus, and facultative anaerobic cocci. Probiotic functions are mainly ascribed to six main aspects: promoting digestion and absorption, enhancing immune cells, protecting intestinal mucosa, curtailing cancer risk, reducing cholesterol absorption, assisting oxidation resistance, and loosening the bowel to relieve constipation $(18,19)$. Prebiotics are food components that cannot be digested or difficult to digest, and these components are beneficial to the health of the host as they selectively stimulate the proliferation and/or activity of bacteria in the colon. Probiotics play a major role in defending against pathogens, regulating immune function, increasing absorption of minerals, improving intestinal function, regulating metabolism, regulating appetite, etc. (20). Several studies have reported that prebiotics (such as fructooligosaccharide an insulin-like fructan) and probiotics (such as Saccharomyces boulardii) can change the composition of intestinal flora, increase the relative abundance of Bifidobacterium and Lactobacillus, and improve glucose tolerance and lipid metabolism (21-23). A study showed that a daily intake of a $200 \mathrm{~mL}$ milkshake containing $4 \times 10^{8} \mathrm{CFU} \cdot 100$ $\mathrm{mL}^{-1}$ Lactobacillus acidophilus, $4 \times 10^{8} \mathrm{CFU} \cdot 100 \mathrm{~mL}^{-1}$ Bifidobacterium, and $10 \mathrm{~g} \cdot \mathrm{L}^{-1}$ fructooligosaccharides can lead to a significant decrease in blood glucose level in patients with T2DM. In another study by the same team, prebiotic supplements for pregnant women with diabetes reduced blood sugar levels during pregnancy and 12 months after delivery, lowered insulin concentrations, and improved insulin sensitivity $(24,25)$. Perraudeau et al. conducted a 12-week intervention in 76 patients with T2DM and found that probiotic supplementation with WBF-011 (containing inulin,

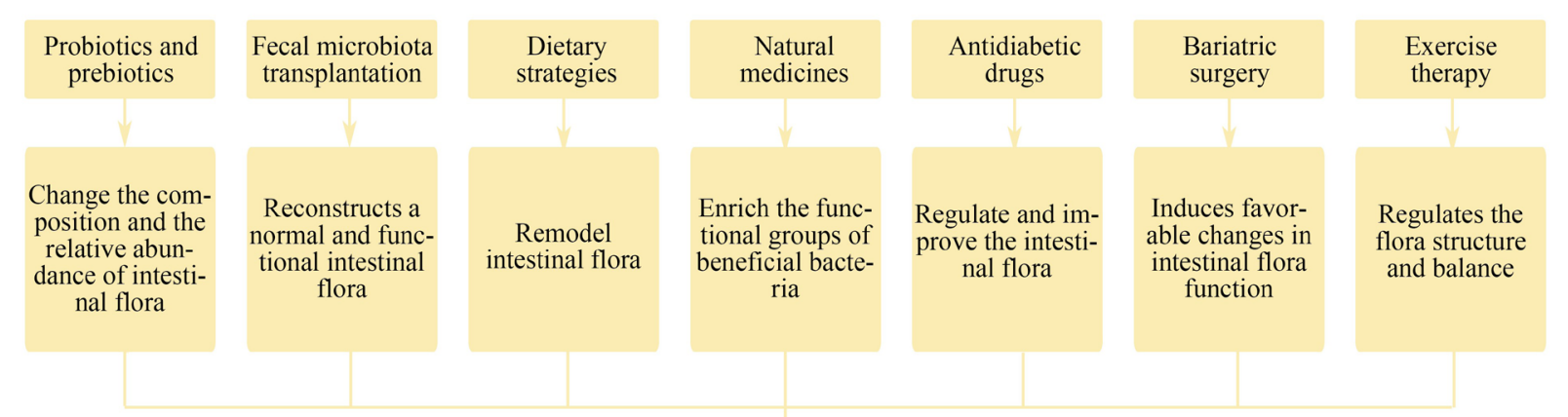

1.Improve glucose tolerance

2.Regulate energy metabolism

3. Regulate bile acid metabolism

4.Prevent insulin resistance

Prevent and treat type 2 diabetes

Figure 1. The possible mechanism of prevention and treatment of type 2 diabetes based on the effect of different strategies on the intestinal flora in various studies. 
Akkermansia muciniphila, Clostridium beijerinckii, Clostridium butyricum, Bifidobacterium infantis, and Anaerobutyricum hallii) can significantly reduce postprandial blood glucose, HbAlc, and incremental glucose area under the curve with good safety and tolerability (26). Rittiphairoj et al. conducted a randomized controlled trial in approximately 2,000 patients with prediabetes or T2DM. The results showed that short-term or long-term probiotic use significantly reduced fasting blood glucose (FBG), HbAlc, and serum total cholesterol (TC). They also reported that probiotics more effectively reduced the HbA1c or FBG levels in patients not receiving insulin therapy (27). It is speculated that appropriate use of probiotics/probiotics can effectively prevent the occurrence and development of T2DM.

\subsection{Fecal microbiota transplantation (FMT)}

FMT has become a research hotspot in the medical field as a new treatment strategy. It is a technical system for treating intestinal diseases or extra-intestinal diseases by transplanting the functional flora from the feces of healthy people into the gastrointestinal tract of patients to reconstruct a normal and functional intestinal flora. FMT can be used to treat Clostridium difficile infection, irritable bowel syndrome, chronic constipation, inflammatory bowel disease, metabolic syndrome (hypertension, diabetes, fatty liver, obesity, etc.), autism, anxiety, depression, tumors, etc. (28). Vrieze et al. and Kootte et al. conducted controlled clinical trials to study whether the intestinal flora of thin people could improve blood glucose and lipid metabolism in men with metabolic syndrome. Six weeks after FMT, subjects who received FMT from thin people had significantly enhanced insulin sensitivity and increased abundance of intestinal flora compared with the control group. The results showed that the number of butyric acidproducing bacteria increased significantly. In addition, the subjects' plasma metabolites (such as $\gamma$-aminobutyric acid [GABA]) change, and butyric acid can regulate energy metabolism and prevent insulin resistance $(29,30)$. Accordingly, FMT may be a good treatment option for patients with T2DM.

\subsection{Dietary strategies}

The major causes of T2DM are weight gain and abnormal visceral fat accumulation. They manifest as a large waistline and lead to metabolic syndrome and multiple complications. The control of obesity is more conducive to treat T2DM than drug intervention, and it can mitigate the progression of the disease at an early stage (31). Therefore, dietary therapy is key to the prevention and treatment of diabetes. Matacchione et al. reported that a diet rich in dietary polyphenols could effectively prevent T2DM by inhibiting the activity of $\alpha$-amylase, $\alpha$-glucosidase, and glucose transporters, stimulating insulin secretion, balancing hepatic glucose, preventing oxidative stress and inflammationrelated hyperglycemia, and remodeling intestinal flora to improve blood glucose. The risk of developing T2DM is reduced by inhibiting or reducing intestinal transport of cholesterol and triglycerides, reducing serum cholesterol, triglyceride, and lipoprotein levels, and interacting with the synthesis and elimination of cholesterol and triglycerides to regulate lipid metabolism (32). Jiang et al. found that higher fruit intake is associated with a lower T2DM risk mediated by specific intestinal flora and metabolites. They found a correlation between fruit intake and the abundance of 31 OTUs belonging to Faecalibacterium prausnitzii, Akkermansia muciniphila, Ruminococcaceae members, Clostridium, Acidaminococcus, Prevotella stercorea, Prevotella copri, Fusobacterium, and Enterobacteriaceae. The fruit-flora index (FMI) was calculated based on the 31 OTUs associated with fruit intake. The FMI negatively correlated with the HbAlc level, and the risk of T2DM decreased by $17 \%$ for each additional unit of the FMI. Fecal metabolite sebacic acid positively associated with the FMI but negatively with T2DM risk. However, several other fecal metabolites negatively associated with the FMI were positively associated with T2DM risk. In a validation cohort of 6626 participants, T2DM risk was reduced by $10 \%$ for every additional unit of the FMI (33). Khursheed et al. reported that polysaccharides in mushrooms could play the role of prebiotics by regulating intestinal flora, metabolizing short-chain fatty acids (SCFAs) to increase the secretion of glucagonlike peptide (GLP)-1, and inhibiting gastric emptying to reduce appetite, thus playing an antidiabetic role (34). Therefore, cultivating good dietary habits (such as increasing the intake of fruits and vegetables) has an obvious effect on the prevention and treatment of T2DM.

\subsection{Natural medicines}

$\mathrm{Xu}$ et al. evaluated the clinical efficacy of different doses of Gegen Qinlian decoction in 187 patients with T2DM. The levels of FBG and HbA1c in patients treated with high and medium doses were significantly lower than those in the placebo and low-dose groups, and the therapeutic effect was dose-dependent. By analyzing the bacterial DNA in fecal samples of the subjects before and after treatment, they found a type of Faecalibacterium prausnitzii, which is closely related to the improvement of diabetes, was significantly enriched in the gut of patients after treatment. This increased bacterial load negatively correlated with levels of $\mathrm{HbAlc}$ and $\mathrm{FBG}$, suggesting that alteration in intestinal bacteria is one of the crucial reasons for improvement in diabetes (35). Tong et al. randomly divided 450 patients with T2DM and hyperlipidemia into metformin and Chinese 
herbal compound (AMC) treatment groups. After 12 weeks of treatment, 100 patients were randomly selected for evaluation of clinical efficacy. The results showed that AMC improved homeostasis model assessment of insulin resistance (HOMA-IR) and plasma triglycerides compared with metformin, and the increase in coenriched bacteria represented by Blautia species was significantly associated with improvement in glycolipid homeostasis, which may improve hyperglycemia and hyperlipidemia by enriching functional groups of beneficial bacteria such as Blautia and Faecalibacterium (36). Tong et al. reported that Chinese herbal medicine and Chinese herbal prescriptions might improve glucose homeostasis and diabetes through the intestinal floramucosal immunity-inflammation-diabetes axis (37). Therefore, AMC may be beneficial to patients with T2DM, through its effect on the intestinal flora.

\subsection{Antidiabetic drugs}

In recent years, studies have found that some commonly used hypoglycemic drugs may regulate and improve intestinal flora in patients with T2DM to some extent, especially metformin (38). Metformin is recommended as a first-line oral drug to control blood glucose levels in patients with T2DM, and a meta-analysis showed that metformin increases abundance of bacteria that produce SCFAs in subjects with T2DM (39). Sun et al. treated patients newly diagnosed with T2DM with metformin and found that composition of intestinal flora changed significantly (Figure 2). Bacteroides fragilis decreased most significantly among other species; and levels of glycoursodeoxycholic acid (GUDCA) and tauroursodeoxycholic acid (TUDCA) in the gut increased. GUDCA and TUDCA are farnesoid X receptor (FXR) antagonists, and metformin increases the GUDCA level by reducing abundance of Bacteroides fragilis to inhibit activity of bile salt hydrolase. Metformin inhibits intestinal FXR signals independently of intestinal adenosine monophosphate-activated protein kinase, significantly increases the production of active GLP-1, and improves glucose homeostasis (40). According to the results of Forslund et al., metformin can improve the intestinal flora of patients with T2DM, promote the production of SCFAs such as butyric acid and propionic acid, stimulate intestinal gluconeogenesis, and increase insulin sensitivity in the body (41). In a clinical trial, 95 patients with T2DM were randomly divided into two groups: group A received acarbose $150 \mathrm{mg} / \mathrm{d}$ and group B received the same treatment as group A but without acarbose. The results showed that compared with group B, intestinal Bifidobacterium increased, and serum lipopolysaccharides and prothrombin activator inhibitor 1 significantly decreased in group A. Acarbose therapy can significantly increase intestinal Bifidobacterium in patients with T2DM and reduce the levels of some inflammatory factors besides

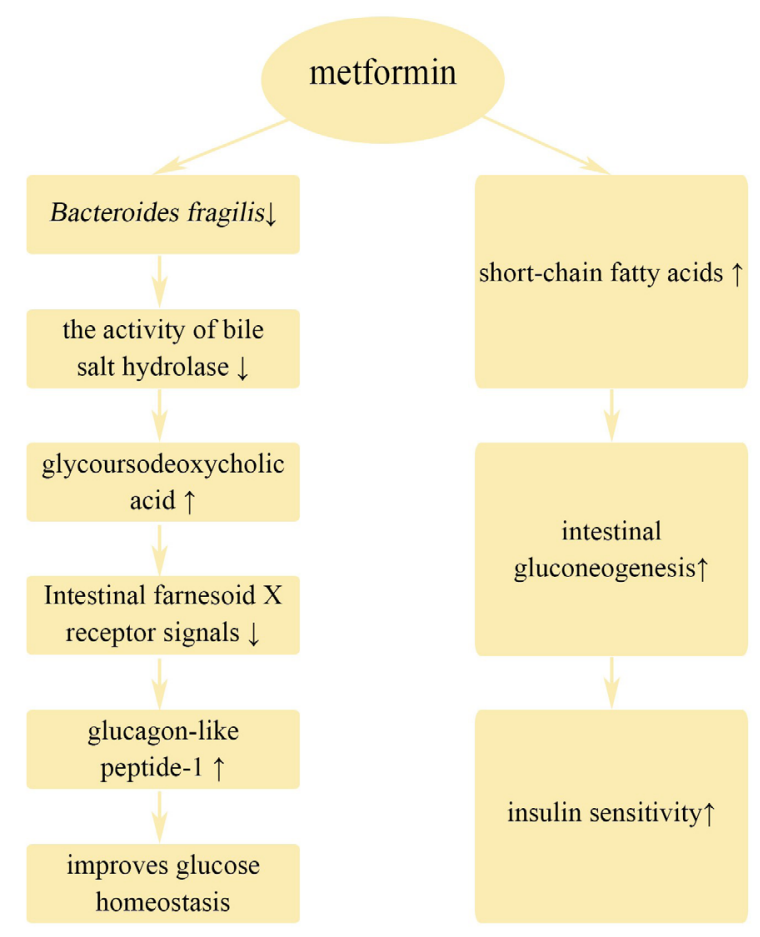

Figure 2. The mechanism of metformin in the treatment of diabetes by regulating intestinal flora. $\uparrow:$ increase; $\downarrow$ :decrease.

reducing blood glucose (42). Gu et al. randomly divided 106 patients with new-onset T2DM into two groups: those treated with acarbose $300 \mathrm{mg} / \mathrm{d}$ or glipizide 5-10 $\mathrm{mg} / \mathrm{d}$ for 3 months. The results showed that acarbose was superior to glipizide in reducing glucose and lipid levels, body weight, and insulin resistance. Treatment with acarbose significantly increased the abundance of various probiotics (such as Bifidobacterium and Lactobacillus) and substantially reduced the abundance of Clostridium and Bacteroides, while there was no significant change in the glipizide group before and after treatment. Acarbose likely regulates glucose and lipid metabolism by changing the bile acid (BA) metabolism of intestinal microbes and affecting the host BA signal, thus achieving benefits other than the hypoglycemic effect (43). Accordingly, when choosing antidiabetic drugs, a more beneficial treatment plan for patients can be developed by taking into account the effects of drugs on intestinal flora.

\subsection{Bariatric surgery}

Linner and Kremen performed the world's first jejunoileal bypass surgery in 1954, pioneering the surgical treatment for obesity. A large number of randomized clinical trials comparing various surgical interventions with non-surgical interventions for diabetes have consistently demonstrated the former's advantage in improving all glucose variables and other metabolic aspects (44). At present, the widely accepted surgical procedures in metabolic and bariatric surgery include 
laparoscopic sleeve gastrectomy (LSG), laparoscopic Roux-en-Y gastric bypass (LRYGB), and biliopancreatic diversion with duodenal switch. Among them, LSG and LRYGB are the most common types of bariatric surgery. For patients with T2DM, bariatric surgery plus medication is more effective than medical therapy alone. Magouliotis et al. found that after metabolic surgery, patients' blood glucose, insulin, triglyceride, TC, low-density lipoprotein, and HDL levels; HOMAIR; food intake; and the rate of diabetes remission were significantly improved. Postoperatively, the levels of BCAA decreased, whereas those of trimethylamine-Noxide (TMAO), GLP-1, GLP-2, and peptide YY (PYY) increased (45). LRYGB and LSG can reduce blood glucose level and body weight in obese patients with T2DM and increase Roseburia species. Compared with LSG, LRYGB can induce more favorable changes in intestinal flora function. LRYGB led to an increase in the abundance of Firmicutes and Actinobacteria phyla and a decrease in Bacteroidetes phyla, whereas LSG led to an increase in the abundance of Bacteroidetes phyla (46). Reports have suggested that bariatric surgery may change the microbiome; furthermore, it has been suggested that intestinal flora may play a role by increasing the absorption of energy in the diet and altering signaling pathways of metabolism and appetite. Clinical studies have shown that circulating BA concentration increases after bariatric surgery, and the total circulating BA concentration in LRYGB patients positively correlates with serum GLP-1 concentration and negatively correlates with postprandial blood glucose concentration. The increased concentration of circulating BA may be due to changes in the anatomical structure and intestinal microbiota caused by bariatric surgery (47). Bariatric surgery may be one of the most effective treatments for T2DM.

\subsection{Exercise therapy}

Exercise therapy in diabetes is mainly suitable for patients with mild and moderate T2DM, especially in obese individuals with T2DM. Exercise can increase the abundance of beneficial intestinal flora and regulate the flora structure and balance. It also significantly enhances the intestinal flora's ability to synthesize SCFAs, decompose BCAAs, and promote secretion of hormone PYY. Furthermore, it inhibits appetite and plays a key role in weight loss, improving insulin resistance, and regulating blood glucose. Low-grade inflammation is a characteristic of T2DM. Moderate running can prevent excessive activation of the immune system, and regular exercise such as endurance training in the form of stationary exercise bikes, aerobics, dumbbell, and other forms, and flexibility training, such as static stretching, can significantly improve the index of glycolipid metabolism and inflammation in patients with T2DM. In addition, the abundance of fungi,
Candida albicans, and mycotoxins can be significantly reduced by exercise without affecting beneficial bacteria such as Lactobacillus and Bifidobacterium (48). Liu et al. randomly divided 39 overweight men with prediabetes into two groups: one group performed 12 weeks of intense exercise and the other group maintained a sedentary lifestyle. After 12 weeks of oneto-one exercise training, all participants had significant and comparable reductions in body weight and body fat percentage without any drug and dietary interventions, while the individual differences in fasting glucose, insulin, and HOMA-IR were significant. Further studies showed that the ability to synthesize SCFAs and GABA and decompose BCAAs was significantly enhanced in the intestinal flora of exercise responders. Whereas, the intestinal flora of non-responders synthesized a large number of products that were not conducive to glucose metabolism, such as BCAAs and aromatic amino acids. These results suggest that intestinal flora and its metabolites mediate improvement in insulin sensitivity and glucose homeostasis by exercise. They are expected to be biomarkers for evaluating and predicting the efficacy of exercise intervention in future studies. Thus, intestinal flora intervention may help maximize the health benefits of exercise (49).

\section{Summary and outlook}

A growing number of studies have shown that intestinal flora is closely related to occurrence and development of T2DM. Intestinal flora may be involved in T2DM pathogenesis via multiple metabolic pathways, such as those mediated by SCFAs, BA metabolism, endotoxin, and TMAO. Regulating intestinal flora by bariatric surgery, probiotics, FMT, exercise, establishing healthy eating habits, antidiabetic drugs and other methods can improve insulin resistance and regulate blood glucose. Although new therapeutic methods are being developed, many uncertainties and potential risks must be accounted for, warranting further investigation.

\section{Acknowledgements}

We thank the participants of this study, including the doctors and researchers from the Department of Endocrinology of The First Affiliated Hospital of Anhui Medical University.

Funding: The Natural Science Foundation of Anhui Province in China (2108085MH269).

Conflict of Interest: The authors have no conflicts of interest to disclose.

\section{References}

1. Saeedi P, Petersohn I, Salpea P, Malanda B, Karuranga 
S, Unwin N, Colagiuri S, Guariguata L, Motala AA, Ogurtsova K, Shaw JE, Bright D, Williams R; IDF Diabetes Atlas Committee. Global and regional diabetes prevalence estimates for 2019 and projections for 2030 and 2045: Results from the International Diabetes Federation Diabetes Atlas, 9th edition. Diabetes Res Clin Pract. 2019; 157:107843.

2. Taddeo EP, Alsabeeh N, Baghdasarian S, et al. Mitochondrial Proton Leak Regulated by Cyclophilin D Elevates Insulin Secretion in Islets at Nonstimulatory Glucose Levels. Diabetes. 2020; 69.

3. Gurung M, Li Z, You H, Rodrigues R, Jump DB, Morgun A, Shulzhenko N. Role of gut microbiota in type 2 diabetes pathophysiology. EBioMedicine. 2020; 51:102590.

4. Junjie Q, Ruiqiang L, Jeroen R, et al. A human gut microbial gene catalogue established by metagenomic sequencing. Nature. 2010; 464:59-65.

5. Zhang X, Shen D, Fang Z, Jie Z, Qiu X, Zhang C, Chen Y, Ji L. Human Gut Microbiota Changes Reveal the Progression of Glucose Intolerance. PloS One. 2013; 8:e71108.

6. Lilit E, Daria K, Anna P, Olga T, Alexander T, Dmitry A, Natalia K, Elena K, Vladislav B, Maria V, Sergey B. Gut microbiota and diet in patients with different glucose tolerance. Endocr Connect. 2016; 5:1-9.

7. Larsen N, Vogensen FK, Berg FWJvd, Nielsen DS, Andreasen AS, Pedersen BK, Al-Soud WA, Sørensen SJ, Hansen LH, Jakobsen M. Gut Microbiota in Human Adults with Type 2 Diabetes Differs from Non-Diabetic Adults. PloS One. 2010; 5:e9085.

8. Wu X, Ma C, Han L, Nawaz M, Gao F, Zhang X, Yu P, Zhao Ca, Li L, Zhou A, Wang J, Moore JE, Millar BC, Xu J. Molecular Characterisation of the Faecal Microbiota in Patients with Type II Diabetes. Curr Microbiol. 2010 ;61:69-78.

9. Karlsson FH, Tremaroli V, Nookaew I, Bergström G, Behre CJ, Fagerberg B, Nielsen J, Bäckhed F. Gut metagenome in European women with normal, impaired and diabetic glucose control. Nature. 2013; 498:99-103.

10. Sedighi M, Razavi S, Navab-Moghadam F, Khamseh ME, Alaei-Shahmiri F, Mehrtash A, Amirmozafari N. Comparison of gut microbiota in adult patients with type 2 diabetes and healthy individuals. Microb Pathog. 2017; 111:362-369.

11. Pedersen HK, Gudmundsdottir V, Nielsen HB, et al. Human gut microbes impact host serum metabolome and insulin sensitivity. Nature. 2016; 535:376-381.

12. Lambeth SM, Carson T, Lowe J, Ramaraj T, Leff JW, Luo L, Bell CJ, Shah VO. Composition, Diversity and Abundance of Gut Microbiome in Prediabetes and Type 2 Diabetes. J Diabetes Obes. 2015; 2:1-7.

13. McLean MH, Dieguez D Jr, Miller LM, Young HA. Does the microbiota play a role in the pathogenesis of autoimmune diseases? Gut. 2015; 64:332-341.

14. Hartstra AV, Bouter KE, Bäckhed F, Nieuwdorp M. Insights into the role of the microbiome in obesity and type 2 diabetes. Diabetes Care. 2015; 38:159-165.

15. Reitmeier S, Kiessling S, Clavel T, et al. Arrhythmic Gut Microbiome Signatures Predict Risk of Type 2 Diabetes. Cell Host Microbe. 2020; 28:258-272.e6.

16. Sroka-Oleksiak A, Młodzińska A, Bulanda M, Salamon D, Major P, Stanek M, Gosiewski T. Metagenomic Analysis of Duodenal Microbiota Reveals a Potential Biomarker of Dysbiosis in the Course of Obesity and Type 2 Diabetes:
A Pilot Study. J Clin Med. 2020; 9:369.

17. McFarland LV. From yaks to yogurt: the history, development, and current use of probiotics. Clin Infect Dis. 2015; 60 Suppl 2:S85-90.

18. Palacios T, Vitetta L, Coulson S, Madigan CD, Denyer GS, Caterson ID. The effect of a novel probiotic on metabolic biomarkers in adults with prediabetes and recently diagnosed type 2 diabetes mellitus: study protocol for a randomized controlled trial. Trials. 2017; 18:7.

19. Henao-Mejia J, Elinav E, Jin C, Hao L, Mehal WZ, Strowig T, Thaiss CA, Kau AL, Eisenbarth SC, Jurczak MJ, Camporez JP, Shulman GI, Gordon JI, Hoffman HM, Flavell RA. Inflammasome-mediated dysbiosis regulates progression of NAFLD and obesity. Nature. 2012; 482:179-185.

20. Sanders ME, Merenstein DJ, Reid G, Gibson GR, Rastall RA. Probiotics and prebiotics in intestinal health and disease: from biology to the clinic. Nat Rev Gastroenterol Hepatol. 2019; 16:605-616.

21. Everard A, Lazarevic V, Derrien M, Girard M, Muccioli GG, Neyrinck AM, Possemiers S, Van Holle A, François P, de Vos WM, Delzenne NM, Schrenzel J, Cani PD. Responses of gut microbiota and glucose and lipid metabolism to prebiotics in genetic obese and dietinduced leptin-resistant mice. Diabetes. 2011; 60:27752786.

22. Everard A, Matamoros S, Geurts L, Delzenne NM, Cani PD. Saccharomyces boulardii administration changes gut microbiota and reduces hepatic steatosis, low-grade inflammation, and fat mass in obese and type 2 diabetic db/db mice. mBio. 2014; 5:e1011-14.

23. Cani PD, Neyrinck AM, Fava F, Knauf C, Burcelin RG, Tuohy KM, Gibson GR, Delzenne NM. Selective increases of bifidobacteria in gut microflora improve high-fat-diet-induced diabetes in mice through a mechanism associated with endotoxaemia. Diabetologia. 2007; 50:2374-2383.

24. Moroti C, Souza Magri LF, de Rezende Costa M, Cavallini DC, Sivieri K. Effect of the consumption of a new symbiotic shake on glycemia and cholesterol levels in elderly people with type 2 diabetes mellitus. Lipids Health Dis. 2012; 11:29.

25. Laitinen K, Poussa T, Isolauri E; Nutrition, Allergy, Mucosal Immunology and Intestinal Microbiota Group. Probiotics and dietary counselling contribute to glucose regulation during and after pregnancy: a randomised controlled trial. Br J Nutr. 2009; 101:1679-1687.

26. Perraudeau F, McMurdie P, Bullard J, et al. Improvements to postprandial glucose control in subjects with type 2 diabetes: a multicenter, double blind, randomized placebo-controlled trial of a novel probiotic formulation. BMJ Open Diabetes Res Care. 2020; 8:e001319.

27. Rittiphairoj T, Pongpirul K, Janchot K, Mueller NT, Li T. Probiotics Contribute to Glycemic Control in Patients with Type 2 Diabetes Mellitus: A Systematic Review and Meta-Analysis. Adv Nutr. 2021;1 2:722-734.

28. Borody TJ, Khoruts A. Fecal microbiota transplantation and emerging applications. Nat Rev Gastroenterol Hepatol. 2011; 9:88-96

29. Kootte RS, Levin E, Salojärvi J, et al. Improvement of Insulin Sensitivity after Lean Donor Feces in Metabolic Syndrome Is Driven by Baseline Intestinal Microbiota Composition. 2017; 26:611-619.e6.

30. Vrieze A, Nood EV, Holleman F, et al. Transfer of 
Intestinal Microbiota From Lean Donors Increases Insulin Sensitivity in Individuals With Metabolic Syndrome. Gastroenterology. 2012; 143:913-916.e7.

31. Lean MEJ. Low-calorie diets in the management of type 2 diabetes mellitus. Nat Rev Endocrinol. 2019; 15:251252.

32. Matacchione G, Gurău F, Baldoni S, Prattichizzo F, Silvestrini A, Giuliani A, Pugnaloni A, Espinosa E, Amenta F, Bonafè M, Procopio AD, Rippo MR, Olivieri F, Sabbatinelli J. Pleiotropic effects of polyphenols on glucose and lipid metabolism: Focus on clinical trials. Ageing Res Rev. 2020; 61:101074.

33. Jiang Z, Sun TY, He Y, et al. Dietary fruit and vegetable intake, gut microbiota, and type 2 diabetes: results from two large human cohort studies. BMC Med. 2020; 18:371.

34. Khursheed R, Singh SK, Wadhwa S, Gulati M, Awasthi A. Therapeutic potential of mushrooms in diabetes mellitus: Role of polysaccharides. Int J Biol Macromol. 2020; 164:1194-1205.

35. Xu J, Lian F, Zhao L, Zhao Y, Chen X, Zhang X, Guo Y, Zhang C, Zhou Q, Xue Z, Pang X, Zhao L, Tong X. Structural modulation of gut microbiota during alleviation of type 2 diabetes with a Chinese herbal formula. ISME J. 2015; 9:552-562.

36. Tong X, Xu J, Lian F, et al. Structural Alteration of Gut Microbiota during the Amelioration of Human Type 2 Diabetes with Hyperlipidemia by Metformin and a Traditional Chinese Herbal Formula: a Multicenter, Randomized, Open Label Clinical Trial. mBio. 2018; 9:e02392-17.

37. Gao Z, Li Q, Wu X, Zhao X, Zhao L, Tong X. New Insights into the Mechanisms of Chinese Herbal Products on Diabetes: A Focus on the "Bacteria-Mucosal Immunity-Inflammation-Diabetes" Axis. J Immunol Res. 2017; 2017:1813086.

38. Morita Y, Nogami M, Sakaguchi K, Okada Y, Hirota Y, Sugawara K, Tamori Y, Zeng F, Murakami T, Ogawa W. Enhanced Release of Glucose Into the Intraluminal Space of the Intestine Associated With Metformin Treatment as Revealed by [18F]Fluorodeoxyglucose PET-MRI. Diabetes Care. 2020; 43:1796-1802.

39. McCreight LJ, Bailey CJ, Pearson ER. Metformin and the gastrointestinal tract. Diabetologia. 2016; 59:426-435.

40. Sun L, Xie C, Wang G, et al. Gut microbiota and intestinal FXR mediate the clinical benefits of metformin. Nat Med. 2018; 24:1919-1929.

41. Forslund K, Hildebrand F, Nielsen T, et al. Disentangling type 2 diabetes and metformin treatment signatures in the human gut microbiota. Nature. 2015; 528:262-266.

42. Su B, Liu H, Li J, Sunli Y, Liu B, Liu D, Zhang P, Meng X. Acarbose treatment affects the serum levels of inflammatory cytokines and the gut content of bifidobacteria in Chinese patients with type 2 diabetes mellitus. J Diabetes. 2015; 7:729-739.

43. Gu Y, Wang X, Li J, et al. Analyses of gut microbiota and plasma bile acids enable stratification of patients for antidiabetic treatment. Nat Commun. 2017; 8:1785.

44. Cummings DE, Rubino F. Metabolic surgery for the treatment of type 2 diabetes in obese individuals. Diabetologia. 2018; 61:257-264.

45. Magouliotis DE, Tasiopoulou VS, Sioka E, Chatedaki C, Zacharoulis D. Impact of Bariatric Surgery on Metabolic and Gut Microbiota Profile: a Systematic Review and Meta-analysis. Obes Surg. 2017; 27:1345-1357.

46. Murphy R, Tsai P, Jüllig M, Liu A, Plank L, Booth M. Differential Changes in Gut Microbiota After Gastric Bypass and Sleeve Gastrectomy Bariatric Surgery Vary According to Diabetes Remission. Obes Surg. 2017; 27:917-925.

47. Kaska L, Sledzinski T, Chomiczewska A, DettlaffPokora A, Swierczynski J. Improved glucose metabolism following bariatric surgery is associated with increased circulating bile acid concentrations and remodeling of the gut microbiome. World J Gastroenterol. 2016; 22:86988719.

48. Pasini E, Corsetti G, Assanelli D, Testa C, Romano C, Dioguardi FS, Aquilani R. Effects of chronic exercise on gut microbiota and intestinal barrier in humans with type 2 diabetes. Minerva Med. 2019; 110:3-11.

49. Liu Y, Wang Y, Ni Y, Cheung CKY, Lam KSL, Wang Y, Xia Z, Ye D, Guo J, Tse MA, Panagiotou G, Xu A. Gut Microbiome Fermentation Determines the Efficacy of Exercise for Diabetes Prevention. Cell Metab. 2020; 31:77-91.e5.

Received July 6, 2021; Revised August 13, 2021; Accepted Septmeber 14, 2021.

${ }^{\S}$ These authors contributed equally to this work.

*Address correspondence to:

Mingwei Chen, Department of Endocrinology, The First Affiliated Hospital of Anhui Medical University, No. 218 Jixi Road, Hefei 230032, Anhui, China.

E-mail:chmw1@163.com

Released online in J-STAGE as advance publication Septmeber 26, 2021. 